\title{
UN ELTRANSLOG SCHLUMBERGER
}

\section{A. Belluigi}

1. - Consideriamo il c.e.m. (campo elettromagnetico) di una sorgente puntiforme di corrente: 0 , elettrodo di dimensioni trascurabili rispetto alla distanza dei punti di misura di tale campo, in un mezzo terrestre omogeneo.

Si suppone con ciò che il ritorno della corrente avvenga a grande distanza dalla sorgente 0 , dimodoché dipartendosi radialmente le linee di flusso da essa, le relative superfici di livello si potranno considerare sferiche concentriche, di centro 0 .

Posto l'elettrodo 0 (mobile) in un foro di sonda, un secondo fisso alla superficie del suolo, le prese di tensione mobili collineari ad 0 lungo l'asse verticale del foro cilindrico, si realizza il classico dispositivo elettrodico di carotaggio elettrico Schlumberger.

Alimentando siffatto circuito a c.c. (corrente continua) d'intensità $I$, se ò la resistività del terreno, si ha, (v. Fig. l), tra i punti $A$ e $B$ la caduta di tensione:

$$
\Delta V=I_{\mathrm{Q}} \cdot M
$$

$M$ è il coefficiente di forma (o geometrico): $4 \pi z_{1} z /\left(z_{2}-z_{1}\right)$ del dispositivo.

La [1] ¿̀ valida, prescindendo dalla resistivita $\varrho_{\mathrm{m}}$ dei fanghi (o considerando $\ell_{\mathrm{m}}=\ell$ ), e dal diametro del foro (o ritenendolo trascurabile rispetto a $z_{1}, z_{2}$ ).

Mentre per i regimi stazionari il campo elettrico vale:

$$
E_{\mathrm{s} .}=-\operatorname{grad} V
$$

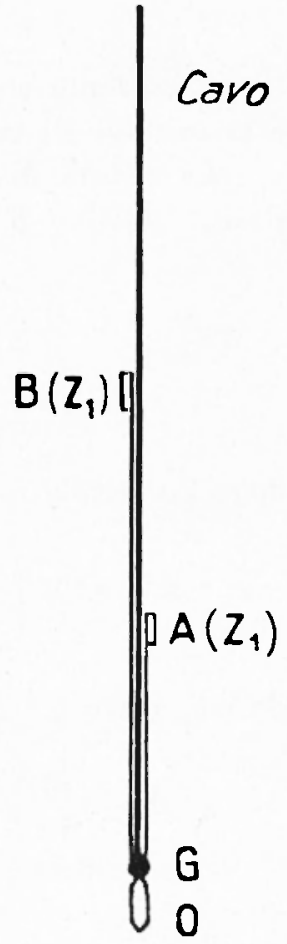

lig. 1

per quelli quasi-stazionari $(v$. [1] $)$, detta $" h \|=(2 ; \sigma(t))^{1 / 2}$ la profondità di penetrazione della corrente nel suolo:

$$
E_{\mathrm{q}, \mathrm{s}}=-e^{-(1+-j) z / \mathrm{h}} \cdot \operatorname{grad} V
$$

che per $(1) \rightarrow 0$, importa ovviamente: $E_{\mathrm{q}, \mathrm{s} .}=E_{8}$ 
2.- Inseriamo ora una step-function di correnle nel precedente circuito tellurico, il campo elettrico s'esprimerà con:

$$
F_{z}(t)=f(t) \cdot I / 4 \pi \sigma z^{2} ; \sigma=e^{-1}
$$

dove $f(t)$ è una cosidetta "funzione transitoria ) (v. [2]), $E_{z}(t)$ la componente verticale del campo elettrico.

Introducendo il tempo: $T=4 \pi \sigma z^{2} \cdot 10^{-9}$, (che dipende dalla strut. tura fisica del terreno e dalla distanza dei punti di osservazione, e assume lo stesso valore su ciascuna delle superfici sferiche potenziali concentriche intorno ad 0 ), la [4] diviene, esplicitando $f(t)$ :

$$
E_{\mathbf{z}}(t)=I \cdot(1-\operatorname{erf} / \bar{T} / \mathrm{H} t) / 4 \pi \sigma z^{2}
$$

È evidente che: $E_{z}(0)=0, E_{z}(\infty)=I / 4 \pi \sigma z^{2}$, qualunque siano $z$ e la costante di tempo: $T(\sigma, z)$.

La caduta di tensione trans tra i punti sulla verticale, asse del pozzo: $A\left(z_{1}\right)$ e $B\left(z_{2}\right)$ sarì pertanto:

$$
\Delta \boldsymbol{V}=\frac{I_{\varrho}}{4 \pi} \cdot \int_{z_{1}}^{z_{2}} z^{-2} \cdot \operatorname{coerf}\left(z \sqrt{\frac{\pi \sigma}{t}}\right) d z
$$

dove l'integrale indefinito in [6] vale, (per l'addendo in ("erf »):

$$
c-z^{-1} \operatorname{erf}\left(\because \sqrt{\frac{\pi \sigma}{t}}\right)+\sqrt{\sigma / t} \lg _{\mathrm{i}} \cdot\left(e^{-\pi \sigma z \approx}\right) ; c=\text { cost. arbitraria, }
$$

da cui, posto $\hat{\rho}=\gamma^{\prime} \bar{\sigma} \sigma / t$ :

$$
\begin{gathered}
\Delta V=\frac{I_{0}}{4 \cdot \tau}\left\{2\left(z_{1}^{-3}-z_{2}^{-3}\right)+z_{2}^{-1} \operatorname{erf} \beta z_{2}-z_{1}^{-1} \operatorname{erf} \beta z_{1}-\right. \\
\left.-\frac{\beta}{\sqrt{\pi}}\left[\lg _{1} e^{-\beta^{2} z_{2}^{2}}-\lg _{1} e^{-\beta^{2} z_{1}^{2}}\right]\right\}
\end{gathered}
$$

I valori estremi di $\Delta V(t)$ risultano:

$$
\Delta V(\infty)=\left(z_{1}^{-3}-z_{2}^{-3}\right) I \varrho / 2 \pi
$$

$$
\Delta V(0)=\left(z_{1}^{-3}-z_{2}^{-3}+z_{2}^{-1}-z_{1}^{-1}\right) I 0 / 4 \pi
$$


La derivata prima rispetto al tempo di $\Delta V(t)$ potendosi scrivere:

$$
\begin{aligned}
& j V(t)=-I ;\left[\lg _{i}\left(,-\xi^{2}\right)-\lg _{\mathrm{i}}\left(e^{-\xi_{1}^{2}}\right)\right] / 4 \pi \sigma \sqrt{\pi} \\
& \xi_{1}=\beta z_{1}, \xi_{2}=\beta z_{2},
\end{aligned}
$$

tenendo conıo che: $-j>0$, il segno di $j V(t)$ dipenderà dalla differenza dei logaritmi integrali.

Posto ora $p(\xi)=\lg _{\mathrm{i}}\left(e^{-\xi^{x}}\right),(p(0)=\lg ,(1)$ o (nell'intervallo: 0,1 , $\left.\lg _{\mathrm{i}} \iota<0\right), \varphi(\infty)-\lg _{\mathrm{i}}(n)=0, \varphi^{1}(\xi)=2 \xi^{-1} e^{-\xi^{2}}>0, \varphi(\xi)$ così è crescente.

Distinguiamo ora 2 casi (il che ha un ovvio significato fisico): $z_{1}<z_{2}, z_{1}>z_{2}$.

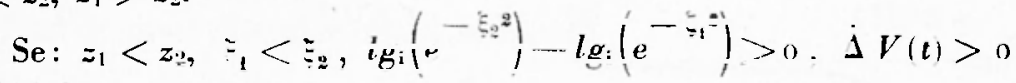

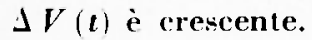

Inoltre: $z_{1}^{-3}-z^{-3}>0, z_{2}^{-1}--z_{1}^{-1}<0$, quindi :

$$
f(0)=\frac{I}{4 \pi \sigma}\left(z_{1}^{-3}-z_{2}^{-3}+z_{2}^{-i}-z_{1}^{-1}\right)<\frac{l}{4 \pi \sigma}\left(z_{1}^{-i}-z_{2}{ }^{-3}=\text { ín } .\right.
$$

Se: $z_{1}>z_{2}, z_{1}{ }^{3}-z_{2}^{3}<0, z_{2}^{-1}-z_{1}^{1}>0$, e quindi :

$$
f(0)>f(\infty)
$$

Inoltre essendo:

$$
\Sigma_{1}>\sum_{2}, \lg \left(e^{-i}\right)-\lg _{i}\left(e^{-3}\right)<0 ; j v(t)<0 ; \Delta V(t)
$$

è decrescente.

Nella fig. 2 i riassunto quanto sopra considerando sia $f(0)>0$, che $f(0)<0$.

3. - Occorre, a questo punto, riconoscere i valori di $f(0), f(\infty) \neq 0$, in funzione della distribuzione tripolare verticale. $E$ ciò ai fini della scelta più conveniente delle geometrie polari che si ripercuotono sul maggiore o minore evidenziamento delle caratteristiche temporali dei geoeltrans cosi attivati. 
a) Indichiamo: $f(0) \cdot 4 \pi \sigma / I=p\left(z_{1}, z_{0}\right)$, ed esaminiamo il segno di a cui sono legati gli eventi di fig. 2 :

$$
\uparrow\left(z_{1}, z_{2}\right)=z_{2}^{-3}(h-1)\left(h^{2}+h+1-z_{2}^{2}\right) ; h=z_{2} z_{1}
$$
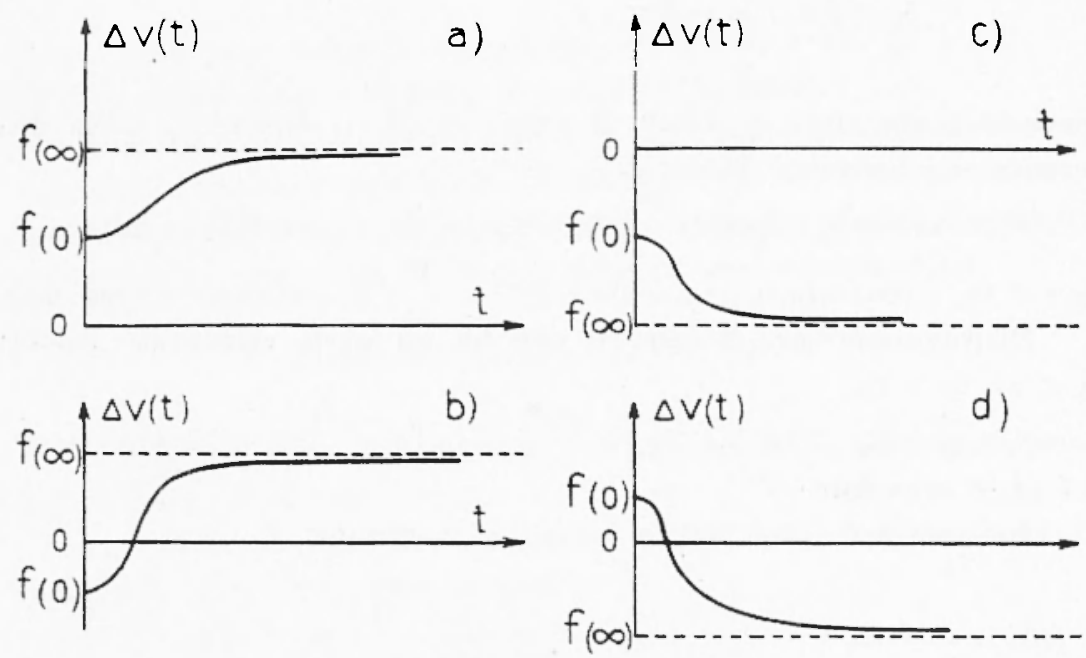

Fig. 2

Dato $z>0, h>0$, il segno di $\varphi_{i}\left(z_{1}, z_{0}\right)$, dipenderà da quello del prodotto a $2^{\circ}$ membro della [12], e questo dai segni di $(h-1)$ e del trinomio $y^{\prime}(h)=\left(h^{2}+h+1-z^{2}\right)$ al variare di $h>0$.

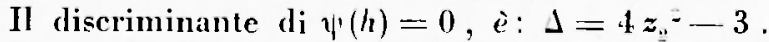

Ora se $\Delta<0, \quad 0<z<<\overline{3} 2$, il trinomio $y^{\prime}|h|$ non si annulla per valori reali di $h$, ed è sempre positivo (per $h=0$ rale: $\left.1-z_{2}{ }^{2}>0\right) ; \quad \mid(h) \geqslant 0$ se $J=0,\left(z_{2}=7 \overline{3} 2\right)$, in quanto si riduce a: $(h+1 / 2)^{2}$ ed è positivo per valori positivi di $h$.

Se invece $\Delta>0,1 / 32<z$ occorre distinguere 2 casi: $13 / 2<z \cdot 2<1 ; 1<z$. Nel 10, $1-z_{1}^{2}>0$, le radici hanno lo stesso segno, ed cssendo la loro somma negativa: $=(-1)$, sono cntrambe negative, e per valori positivi di $h$ il trinomio $\dot{e}$ ancora positivo. Se invece $z>1$, esiste una radice positiva: $\bar{h}=0.5\left(-1+\sqrt{4 z^{2}-3}\right)$, e per: $0<h<\bar{h}, \psi^{\prime}(h) \grave{c}$ negativo, per $h=\bar{h}$ vale zero, per $\bar{h}<h$ è positivo.

Prospettiamo tali eventualita come segue nel quadretto sottostante: 


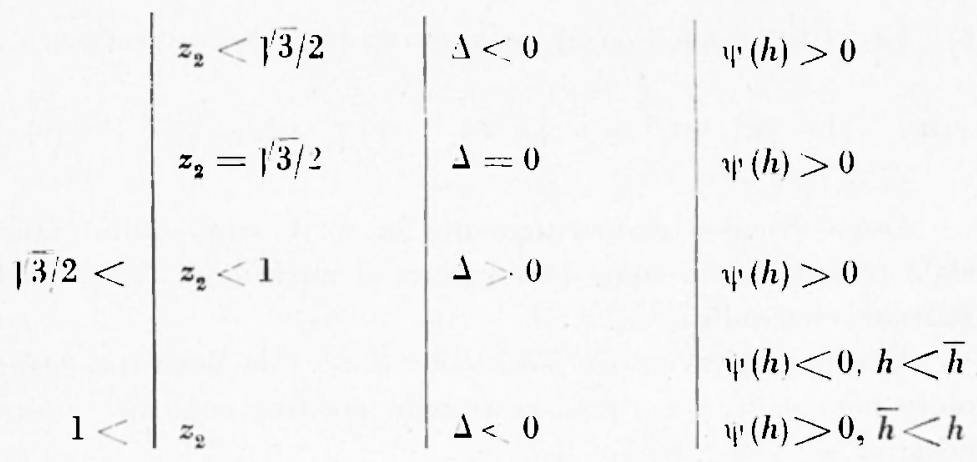

Nei primi 3 casi il serno di $\{(z, z) \mid$ dipende evidentemente da quello di $(h-1)$, ed è negativo per $h<1$, (cioè se $z_{2}<z_{1}$ ), e positivo per $h>0$ (cioè se $z_{2}>z_{1}$ ).

Nel 4" caso, distinguiamo 3 sottocasi:

$1^{\circ)} \bar{h}<1$. Allowa se $h<\bar{h}, h-1<0, y^{\prime}(h)<0$, (f $\left(z_{1}, z_{0}\right)>0$;

$$
\begin{aligned}
& \text { s. } \bar{h}<h<1, h-1<0, \forall(h)=0, \text { i }\left(z_{1}, z_{2}\right)<0 \text {; } \\
& \text { se } 1<\bar{h}, h-1>0, \varphi(h)>0, \varphi\left(z_{1}, z_{2}\right)>0 .
\end{aligned}
$$

20) $\bar{h}=1 . \therefore h<\bar{t}<1, h-1<0, \psi^{\prime}(h)<0, \varphi\left(z_{1}, z_{2}\right)>0$; se $h<h, h-1>0, \psi(i)>0, \varphi\left(z_{1}, z_{2}\right)>0$.

$3^{\text {) }) ~} \bar{h}>1 . \therefore \mathrm{e} h<1<\bar{h}, h-1<0, \psi(h)<0$, $\left(z_{1}, z_{0}\right)>0$; se $\bar{h}<h, h-1>0$. $(h)>0, \psi\left(z_{1}, z_{2}\right)>0$.

Riassumiamo quanto sopra nel seguente quadro, che permette di poter slabilire le condizioni, (v. fig. 2 , per cui: $f(0) \lessgtr 0$.

$$
\begin{aligned}
& h<h \quad, \quad q\left(z_{1}, z_{2}\right)>0 \\
& \bar{h}<1 \quad \bar{h}<h<1, \quad \varphi\left(z_{1}, z_{2}\right)<0 \\
& 1<h \quad, \quad \varphi\left(z_{1}, z_{0}\right)>0 \\
& \bar{h}=1 \quad \text { ( }\left(z_{1}, z_{z}\right)>0 \\
& h<1 \quad, \quad \uparrow\left(z_{1}, z_{1}\right)>0 \\
& \bar{h}>1 \quad 1<h<\bar{h}, \quad \text { if }\left(z_{1}, z_{.1}\right)<0 \\
& \bar{h}<h \quad, \quad \varphi\left(z_{1}, z_{2}\right)>0
\end{aligned}
$$


b) La : $f(0) 2 \pi \sigma / I=\varphi(m)$, con $m=z_{1} / z_{\mathrm{g}}=h^{-1}$ si serive :

$\varphi(m)=\left[1-m^{3}+z_{1}{ }^{2}(m-1)\right] / 2 z_{1}{ }^{3}, \varphi( \pm \infty)=+\infty, \varphi(0)=\frac{1-z_{1}{ }^{2}}{2 z_{1}{ }^{2}}$

Dopo discusso esaurientemente in a) il segno della funzione iniziale $f(0)$, vediamo come può variare il modulo di $f(0)$, con la distribuzione elettrodica.

Poiché: $\quad \varphi^{\prime}(m)=\left(-3 m^{2}+z_{1}{ }^{2}\right) / 2 z_{1}^{3}$ tale derivata si annulla intanto per: $m=+z_{1} / / \overline{3}$, risultando positiva se $m /<z_{1} / \sqrt{3}$, negativa se $|\boldsymbol{m}|>z_{1} / \bar{\gamma}$.

Se ne deduce che: $\varphi(m)$ è decrescente nell'intervallo $(-\infty$, $\left.-z_{1} / \sqrt{3}\right)$, crescente nell'intervallo $\left(-z / \overline{3}, z_{1} / \sqrt{3}\right)$, decrescente nell'intervallo $\left(z_{1} / \sqrt{3},+\infty\right)$, ed è dotata di minimo relativo nel punto $-z_{1} / \gamma \overline{3}$, di massimo relativo nel punto $z_{1} / \gamma^{/} \overline{3}$. Inoltre la \& $(\mathrm{m})$ si annulla per: $m=1 ;-1 / 2-\sqrt{z_{1}^{2}-3 / 4} ;-1 / 2+\sqrt{z_{1}^{2}-3} / 4$, (con $z_{1}>\sqrt{3} / 2$ per la realtà delle radici).

Ciò posto distinguiamo le seguenti situazioni :

$$
0<z_{1}<\sqrt{3} / 2, \quad 1 / 3 / 2<z_{1}<1, \quad 1<z_{1}<\sqrt{3}, \quad z_{1}>\sqrt{3}
$$

$\left.1^{\circ}\right) 0<z_{1}<\sqrt{3} 2$. Osserviamo che $z_{1}<1, z_{1}<\sqrt{3}$, si ha qf $(0)>0, z_{1} / \overline{3}<1,, z_{1}^{2}-3 / 4<0$, quindi $\varphi(m)=0$ ha la radice reale $m=1$ e la funzione $(m)$ consegue il massimo relativo per un valore di $m<1$. Il valore minimo relativo (conseguito per $m=-z_{1} / \sqrt{3}$ è necessariamente positivo, non potendo la funzione annullarsi fuorché per $m=1$.

$2^{\circ)} \gamma^{\prime} \overline{3} / 2<z_{1}<1$. Si ha: $\uparrow(0)>0, z_{1} / \sqrt{3}<1, z_{1}{ }^{2}-3 / 1>0$, onde le 3 radici di $q(m)=0$ sono reali; anzi esclusa la radice $m=1$, le altre 2 sono negative.

$$
\left.3^{\circ}\right) 1<z_{1}<\sqrt{3} \text {. Si ha: } 1(0)<0, z_{1} / \mid \overline{3}<1, z_{1}{ }^{2}-3 / 4>0 \text {, tutte }
$$
e tre le radici di $\uparrow(m)=0$ reali. Il caso differisce dal precedente perché qui 2 radici reali sono positive e l'altra negativa.

$\left.4^{\prime \prime}\right) z_{1}>\sqrt{3}$. Si ha: $q(0)<0, z_{1}>\sqrt{3}, z_{1}{ }^{2}-3 / 1>0$ : tre radici reali, di cui una maggiore dell'unità.

Si osservi che, in tutti i casi, massimo e minimo relativi valgono:

$$
\begin{aligned}
& \uparrow\left(z_{1} / \sqrt{3}\right)=!\left(z_{1}\right)_{\max }=\left(1+2 z_{1}^{3} / 3 \sqrt{3}-z_{1}^{2}\right) 2 z_{1}^{3} \\
& 4\left(-z_{1} / \sqrt{3}\right)=!\left(z_{1}\right)_{\min }=\left(1-2 z_{1}^{3} / 3 \sqrt{3}-z_{1}^{2}\right) / 2 z_{1}^{3}
\end{aligned}
$$


Il luogo di massimi (Fig. 3) permetterà la scelta più favorevole delle $z_{1}$, per $z_{1}<<\sqrt{3}$, mentre nell'intorno di $z_{1}=\sqrt{3}$ si trovano le situazioni più sfavorevoli,

Per valori di $z_{1}>>\sqrt{3}$ la $\|\left(z_{1}\right)$ rimane invariata per quanto si aumenti $z_{1}$ :

infatti: $u(\infty)=1 / 3 \mid \overline{3}, u(0)=\infty, \mu^{\prime}(z)=\left(z_{1}{ }^{2}-3\right) / 6 z_{1}^{4}$, che si annulla per $z_{1}=\sqrt{3}$, e positiva se $z_{1}>\sqrt{3}$, negativa se $z_{1}<\sqrt{3}$, onde и $\left(z_{1}\right)$ decresce Ira 0 e $\sqrt{3}$, cresce da $/ \overline{3}$ a $+\infty$, ha un minimo per $z_{1}=\mid \overline{3}$, per cui : $\mu(\mid \overline{3})=0$.

c) Posto: $z_{1 / 2} / z_{2}=m$,

$$
f(0) / f(c \circ)=!\left(m, z_{1}\right)=0,5\left[1-z_{1}{ }^{2} /\left(m^{2}+m+1\right)\right]
$$

che varia al variare di $\mathrm{m},\left(z_{1}\right.$, parametro $)$.

Gli estremi valgono: $\eta^{\prime}\left(0, z_{1}\right)=0,5\left(1-z_{1}^{2}\right) ; \psi^{\prime}\left(-\infty, z_{1}\right)=0,5$, mentre $u \prime^{\prime}(\mathbf{m})=z_{1}{ }^{-}(2 m+1) 2\left(m^{2}+m+1\right)^{2}$, s'annulla se $m=-1 / 2$, i positiva se $m>-\frac{1}{2}$, negativa se $m<-1 / 2$, onde per $m=-1 / 2$, la funzione presenta un minimo relativo che $i$ anche assoluto:

$$
\psi_{\min }=\left(3-4 z_{1}^{2}\right) / 6
$$



Fig. 3

Ma: $\left.\|^{\prime}(m)=m^{-}+1+z^{2}\right), 2\left(m^{2}+m+1\right)$, si annulla altresì per $\boldsymbol{m}=0,5\left(-1+14 z_{1}^{2}-3\right)$, radici reali se $/ z_{1}>1 \overline{3} / 2,\left(z_{1}{ }^{2}-3 / 4>0\right)$, complesse se $/ z_{1}|<| \overline{3} / 2,\left(z_{1}{ }^{2}-3 / 4<0\right)$. Nel $1^{\text {" }}$ caso si vede subito che si ha: $\mid\left(---\frac{1}{2}, z_{1}\right)<0$, nel $\left.2^{\circ}, 1 /-\frac{1}{2}, z_{1}\right)>0$ : l'andamento della funzione è del tipo:
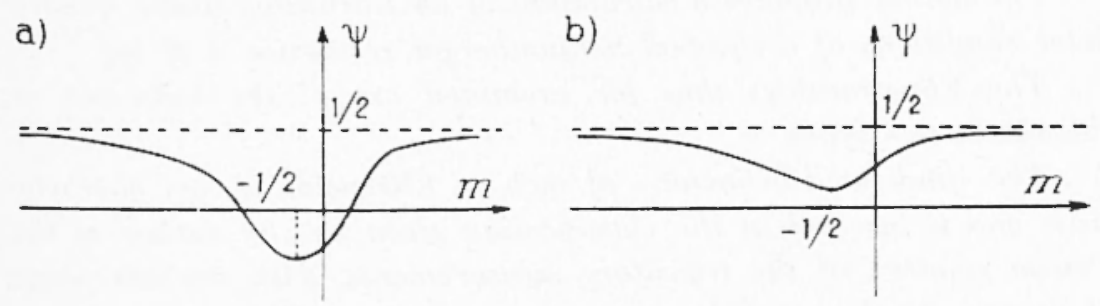

Fig. 4 
Si conclude che quest'( Eltranslog Schlumberger ) rientra in una categoria grià da noi illustrata di geoeltrans (1) di "medio rendi. mento ") agrli effetti indicativi del parametro resistività elettrica.

A parte la nota comune a tuti gli Elmatranslog, di un decorso temporale di misura " tensione trans", in questo procedimento appare particolarmente caratteristico, l'inizio del decorso temporale, funzione $(\neq 0$ ) del valore di regime, della resistività del mezzo e della geometria del dispositivo tripolare.

Ciò trova stretta analogia con ciò che abbiamo chiamato "prima relazione fondamentale " dei geoeltrans $(")$, e con un lipo particolare di Matransloy ("), particolarmente da noi illustrato.

Cagliari - Facoltà d'Ingegneria - Novembre 195.3.

\section{RIASSUNTO}

L'Autore si è proposto il calcolo d'un Eltranslog nelle condizioni quadripolari d'un classico carotaggio resistivo Schlumberger.

Gli Elmatranslog finora esaminati sono a carattere induttivo o galvanico-induttivo.

Il "rendimento indicativo" di tale Eltranslog è ben caratteristico, - s'inserisce nella classifica fatta dell'autore dei "rendimenti medi ", dei carotaggi transitori, col vantaggio di una maggior semplicità realizzatrice della corrispondente apparecchiatura.

\section{SUMMARY}

The author proposes a calculation of an Eltranslog under quadripolar conditions of a classical Schlumberger resistence well log.

The Elmatranslogs thus far examined are of the inductive or galva-indactive types.

The "indicated behavior" of such an Eltranslog is very characteristic and is inserted in the classification given by the author of the "mean results" of the transitory measurements, with the advantag" of greater simplicity of the corresponding apparatus. 


\section{BIBLIOGRAFIA}

(1) J. Kotnigsbemin, Das magnetische Feld einer stromquelle in Raum I'hy. sik. Zeit. 1927).

A. Petnowski, Bulletin of Practical Geopliysies, Leningrad 1928, n. 4.

S. S. Stefanescit. Etudes théoriques sur la prospection du sous-sol, $1^{0}$ partie. Bucuresti 1929.

1. BELLUIGI, Berechnang des magnetischen Feldes einer störung in elektrisch durch stromtem Boden 1933. Ergänz. f. ang. Geoph.

-, Curve universali per il calcolo delle influenze e.m. Boll. Uff. Geol. d'Ita. lia 1946.

-, Campi e.m. dei suoli galvanicamente elettrizzati. Pubbl. Facoltà d'Ingegneria Mineraria di Cagliari 1946, n. 11.

-, LElettrologin galvanica nei conduttori tridimensionali (suoli). Boll. Assoc. Mineraria Sarda, 1948.

(2) A. Beluvigr, Fondamenti teorici dei Geoeltrans. Boll. del serv. Geol. d'Italia, 1952, v. LXXIV.

(3) A. Belcutgr, Lineamenti teorici di nuovi $m$. di carotaggio fisico: Matranslog. Phaselog. Geofisica Pura e Appl., V, 25, 1953. 